(c) Elsevier/INRA

Forum

\title{
Mobilizing selection as a factor in macroevolution
}

\author{
VA Berdnikov*, OE Kosterin \\ Institute of Cytology and Genetics of the Russian Academy of Sciences, \\ Laboratory of Experimental Modelling of Evolutionary Processes, \\ Siberian Department, Academician Lavrentiev Avenue, 10 , \\ Novosibirsk, 90, 630090 Russia
}

(Received 20 November 1990; accepted 23 June 1993)

\begin{abstract}
Summary - We hypothesize that the probability of phyletic lineages surviving under prolonged environmental change depends on the mobility of the working structures of an organism, ie on a genetically determined ability to change structures under the pressure of natural selection. If survival of phyletic lineages regularly depends on the change of the same working structure, mobilizing selection should act on phyletic lineages and favour an increased mobility of this structure. The mobility of a structure increases in proportion to the number of genes governing its formation. As each gene contributes to the enhancement of the structure, the trend for an increase in mobility is manifested as a macroevolutionary trend for an increase in size, power and complexity of the structure. Thus, the progressive development of structures is a result of the increase in their mobility. A computer simulation of the evolution of a quantitative trait controlled by a variable number of genes from a constant pool was carried out with the probability of extinction depending on the rate of favourable mutations. A gradually diminishing increase in the mean and the standard deviation of the trait, accompanied by an increase in the number of genes implied, was observed up to achievement of a stationary distribution. This concept is supported by the evolution of the septal suture of Ammonoidea. This process is characterized by a simultaneous increase in the mean value and standard deviation of suture complexity. This process gradually decelerated and ceased in the early Jurassic.
\end{abstract}

macroevolution / evolutionary progress / computer simulation / Ammonoidea / septal suture

Résumé - La sélection mobilisatrice comme facteur de macroévolution. Nous avançons l'hypothèse que la probabilité de survie d'un phylum dans des conditions de milieu changeantes sur une longue période dépend de la mobilité des structures de fonctionnement de l'organisme, c'est-à-dire d'une aptitude, déterminée génétiquement, à changer les structures sous la pression de la sélection naturelle. Si cette survie du phylum dépend régulièrement du changement de la même structure, la sélection mobilisatrice doit agir

\footnotetext{
${ }^{*}$ Correspondence and reprints
} 
en faveur d'une mobilité accrue de la structure. Cette mobilité augmente proportionnellement au nombre de gènes gouvernant sa formation. Comme chaque gène contribue au renforcement de la structure, la tendance vers une mobilité accrue se manifeste par une tendance macroévolutive vers une augmentation de la taille, de la puissance et de la complexité de la structure. Une simulation par ordinateur a été réalisée pour un caractère quantitatif contrôlé par un nombre variable de gènes à partir d'un réservoir total constant, avec une probabilité d'extinction dépendant du taux des mutations favorables. On observe une augmentation graduellement décroissante de la moyenne et de l'écart-type du caractère, accompagnée d'une augmentation du nombre de gènes impliqués, qui aboutit à une distribution stationnaire. Ce concept est illustré par l'évolution des sutures septales des Ammonites. Ce processus est caractérisé par un accroissement simultané de la valeur moyenne et de la variabilité de la complexité des sutures. Cet accroissement a subi une décélération progressive pour cesser complètement au début de l'ère jurassique.

macroévolution / progrès évolutif / simulation par ordinateur / Ammonites / suture septale

From the Darwinian point of view, the phenomenon of so-called progressive macroevolutionary trends is commonly explained as a result of orthoselection, which implies fundamental advantages from the high complexity, size and power of certain organs in a variety of environments. However, each case has equally convincing counter-arguments. Speculations on the advantages of a large or small body size when explaining the well-known Cope's rule (Stanley, 1973; Grant, 1985; SchmidtNielsen, 1981) serve as a good example.

This work illustrates that many cases of evolutionary progress can be explained without the idea of orthoselection (for details, see Berdnikov, 1990, 1991).

\section{THE CONCEPT OF MOBILIZING SELECTION}

\section{Special genes}

It is well-known that every supercellular structure of an organism performing any biological function (termed a working structure in this text) is formed during ontogenesis according to a certain genetic programme. The genes governing the: development of a working structure will be termed the special genes of the structure. Each special gene expressed brings a particular contribution to the formation of the structure. So, for any species, the development of a working structure is determined by the number of special genes and by the level of their expression. If no special gene is expressed, a complete reduction, or absence, of the structure should be observed, whereas the maximal structure development should reflect the total number of special genes. Let us consider a group of related taxa differing by the number of special genes of a certain structure, where the species comprising each taxon possess the same number of special genes. In such a case the development of the structure within a taxon can vary owing to the interspecies differences in the level of expression of the special genes. Provided that the mean level of their expression is independent of their number, the mean degree of the structure development within a taxon should depend directly upon the number of special genes. 
Thus, we can hypothesize that progressive development of working structures is accompanied by accumulation of special genes. Hence, evolutionary progress should lead to an increase in the informational contents of a genome. However, such a trend would be hindered by an increase in mutational genetic load. Indeed, the mean rates of spontaneous mutation with a qualitative effect in various plants and animals per locus and per generation are quite similar (Sturtevant, 1965). The amount of genetic information seems to be maintained at such a level that the total number of spontaneous mutations per gamete does not exceed a certain value, (Crow and Simmons, 1983; Kondrashov, 1988).

\section{Mobility}

It is important to point out that many environmental factors are subjected to fairly long-term fluctuations with relaxation times ranging from hundreds to thousands or even millions of years, as seen in orogenic cycles, fluctuations of the ocean level, climatic changes, appearance of new predators, etc. As a result many niches disappear and the species occupying them face 2 alternatives: to either become extinct or occupy new niches by transformation into new species. Such a mode of environmental change will be termed a slow catastrophe. On the microevolutionary level, a slow catastrophe means a long-acting natural selection directed to compensate for unfavourable changes. A unidirectional and long-term mode of slow catastrophes enables natural selection to cause speciation events. Note that on the geological time-scale slow catastrophes can appear quite quickly and result in a punctuated speciation pattern.

We suppose that surviving phyletic lineages, having undergone hundreds of slow catastrophes, differ from extinct ones by increased adaptability, ie the ability to overcome slow catastrophes. If adaptability is genetically determined (ie transmitted from an ancestor species to its descendant, and subject to variability), there should be a trend for increased adaptability.

It is evident that deterioration of habitat results in a decrease in species biomass. This is a consequence of some working structures becoming ineffective. Therefore, species survival demands an increase in the functional capacity of this limiting structure. If, in a new environment, the intensity of the function of a structure becomes excessive, the reduction of this structure will leave spare energy that could be used for additional biomass production.

Therefore, the probability of species survival may depend on the maximal rate at which limiting structures can increase or decrease their power under the pressure of selection. The ease of such evolutionary changes in a working structure is termed mobility.

Structure mobility is obviously associated with the rate of change in function due to mutations fixed by natural selection. This rate depends on at least 3 factors: the size of an evolving population; the mutability of a structure (the mean rate of mutations affecting the structure per genome per generation); and the exposure or favourable mutations to selection, ie their selection coefficient values (Kimura, 1983). The relationship between population size and mobilities of particular structures can hardly be imagined. It is also evident that mutability of a structure is directly related to the number of its special genes and their mean mutability. We 
can, therefore, conclude that there is a way of increasing the mobility of a structure by increasing the number of its special genes. This is only true in the absence of a negative correlation between the number of special genes and their exposure to selection. The following considerations suggest that this correlation does not usually take place.

- First, it is well known that for any quantitative trait, including the parameters of a working structure, if the trait is measured on a logarithmic scale its inheritance usually fits an additive multifactor model well (Mather and Jinks, 1982). In this model each allelic substitution affects a quantitative trait independently of the number and allelic state of the other genes. This means that an allelic substitution multiplies the trait value measured on a natural scale by a certain factor. Such an inheritance mode results from integration of special genes into a developmental program with consequent action of genes during ontogenesis.

Secondly, the standard deviation is usually proportional to the mean in both natural populations and pure lines (implying that the traits have logarithmically normal distributions). In the latter case the standard deviation is a measure of the non-heritable variation of the trait (Giller, 1904; Wright, 1984). To allow for selection, the effect of a mutation must be at least comparable with non-heritable variation of the selected trait, $i e$ be proportional to the trait value. Therefore, it is natural to suppose that selection measures traits on a logarithmic scale, where each allelic substitution affects a trait independently of the other genes. Thus, each special gene of a working structure seems to be equally exposed to selection.

\section{Mobilizing selection}

If the survival of a phyletic lineage is regularly determined by changes in the same limiting structure, a natural selection acting upon individuals will cause (as a macroevolutionary consequence) a trend of increased mobility of this structure. Therefore, mobilizing selection occurs among phyletic lineages occupying the same adaptive zone.

We concluded above that the mobility of a working structure can be increased by accumulation of its special genes. This in turn leads to increased mean values for the size, complexity, and power of the structure among species of the evolving clade. Therefore, the morphological progress of a structure turns out to be a mere. consequence of increasing its mobility. Note that natural selection can lack a prevailing direction in a long series of slow catastrophes. Nevertheless, morphologic progress continues.

An accumulation of special genes for any limiting structure does not imply that morphologic progress proceeds in all phyletic lineages of an evolving clade. The clade could contain species with a poorly developed structure due to the low expression of its special genes.

Creation of genes with a new biochemical function seems to be a highly improbable event. To obtain new special genes, existing ones can be altered in two ways: i) rearrangement of regulatory sites leading to change in tissue specificity and/or developmental regulation; and ii) duplication of a gene with further functional divergence accompanied by changes in regulatory sites. In both cases, evolution can use the slight pleiotropic effects of genes for a limiting structure . 
through changes in their controlling regions, with the biochemical function of these genes being, as a rule, retained.

Duplication of genes has, however, a serious drawback: an increase in the informational content of the genome and consequently of mutational genetic load. This seems to be compensated for by the loss of some genes controlling structures whose functions have become excessive in new environments.

Historically, the first source of special genes may have been the genes of intracellular functions (house-keeping genes). Practically every gene involved in the control of complex organs displays a striking similarity with some of the housekeeping genes. An excellent example is provided by the lens crystallines (Wistow and Piatigorsky, 1987).

\section{The principle of maximal adaptability}

Taking into account the enormous evolutionary age of phyletic lineages, we suggest that their adaptability tends to become maximal. The limitation on the volume of genetic information implies that accumulation of a large number of special genes of a certain structure can only be achieved at the expense of genetic maintenance of other structures. Therefore, an equilibrium between the mobilities of working structures should be established when the level of mobility of every structure corresponds to the frequency of its being limiting in a long series of slow catastrophes. It is evident that such an equilibrium provides phyletic lineages with maximal adaptability in their adaptive zone.

As soon as the equilibrium is established, the progressive advancement of any structure should cease. Retardation and cessation of morphological progress is observed in the palaeontological record of many groups of organisms, eg, aquatic arthropods (Cisne, 1974), dipnoan fishes (Simpson, 1953) and Ammonoidea (see below).

Let us consider 2 conventional types of adaptive zones, simple and complex, differing in the number of fluctuating factors. In simple zones, the environment demands the mobility of a small number of structures. These structures undergo progressive development by accumulating a large number of special genes, while the other structures are left for reduction, eg, the degradation of many structures in parasites, with extremely complicated reproductive structures. In complex adaptive zones, the environment demands a larger number of functions. Mobilizing selection is not able to provide every function with sufficient genetic maintenance and all structures appear as non-specialized.

In a situation where structures compete for a genetic resource, a solution can be found in a small number of polyfunctional structures ( $e g$, the central nervous system, limbs of arthropods). This allows the accumulation of a large number of genes to determine few structures. Indeed, this seems to underlie many evolutionary breakthroughs leading to new higher taxa. 


\section{A NUMERIAL MODEL FOR MOBILIZING SELECTION}

\section{The principles of the model}

We have modelled the evolution of a phyletic lineage as a chain of species which possess a quantitative character, $e g$, any parameter of a working structure measured on a logarithmic scale. The value of the character is determined by the additive contributions of the special genes. Allelic substitutions in these genes can change the value of the character. The species genome comprises a constant number of genes, each of which may be either a special or non-special gene. Gene transitions of special to non-special genes and vice versa are allowed.

We assume that any character value should satisfy the demands of the environment. To introduce environmental fluctuations, a random variable is used whose distribution determines the probability of the demanded shifts. The features of the variable are as follows: i) zero expectation, ie the lack of a preferable direction for environmental changes (absence of orthoselection); and ii) identical distribution and statistical independence of environmental fluctuations in all subsequent steps of the model. These requirements are not always met in nature, but in our model we attempt to simulate the most random regime of environmental change.

Each environmental change lasts for the same time interval. In response, the phyletic lineage has to change the value of the character to the required extent during the interval. If the change has not taken place, or the capabilities of the genetic system for further changes are exhausted, the lineage becomes extinct. The extinction probability depends on the extent of the environmental change and on the mobility of the character, determined by the number of special genes.

It is evident that the phyletic lineage eventually becomes extinct. However, in reality the extinction of lineages is compensated by the branching of others. The model ignores branching, but an evolving clade may be regarded as a statistical ensemble of phyletic lineages which are analogous to the model if we assume that: i) branching goes on independently of the character changes, constantly providing a large number of lineages; and ii) the character changes in different phyletic lineages are statistically independent. In such a clade the distribution of the character value is equivalent to the distribution of the conditional probabilities of the modelled phyletic line having certain character values provided that it is not extinct. The same is true for the distribution of the number of special genes. The aim of the model is to study the dynamics of these distributions for a phyletic lineage originating from a founder species with the minimal character value and special gene number.

\section{Description of the model}

The genome contains a constant total number of genes, $G$, of which $N$ represents the number of special genes of the character. Each special gene can be in 1 of the 2 allelic states: 0 or 1 . The character value $Z$ is equal to the number of special genes in the allelic state 1.

Our model is the first-order Markovian chain, which describes a random walk over the range of values of random integer variables $Z$ (character value) and $N$ (number of special genes) where possible states are limited by the conditions $0 \leqslant Z \leqslant N$ 
and $0 \leqslant N \leqslant G$. The set of states is supplemented by the absorptional state $(E)$ corresponding to the extinction of the phyletic lineage. The distribution of the probabilities is investigated for the system in different states $(N, Z)$ provided that it is not in state $(E)$. At each step of the model, these probabilities are calculated directly with subsequent normalizing to unity.

In each step, the character value $Z$ is supposed to change by $\Delta Z . \Delta Z$ is determined as a random integer variable with the range of variation $-R \leqslant \Delta Z \leqslant R$ and a symmetrical binomial distribution of probabilities $P_{1}(\Delta Z)$, where $R$ is a positive integer parameter. (Note that the value of $P_{1}$ is maximal at $\Delta Z=0$.)

If $\Delta Z=0$, the phyletic lineage should fix a series of mutations within a unit of macroevolutionary time $\tau$, in order to provide a character shift by the required value of $\Delta Z$ and, hence, to survive. The probability of such an event depends on the number of genes that can mutate in a proper direction, and on their mutability. Favourable mutations can be associated with either: i) a change in the number of special genes; or ii) the change in the allelic state of those genes. If the character value is to increase, the donors of favourable mutations of type i are $G-N$ nonspecial genes, and those of type ii are $N-Z$ special genes in allelic state 0 . If a decrease in the character value is required, the donors of mutations of both types are $Z$ special genes in allelic state 1 . These genes may either turn into non-special genes or change their allelic state to 0 .

Reaching a required character value is achieved through 2 consecutive stage. First, the character value changes owing to a change in the number of special genes by $\Delta N$ with the probability $P_{2}(\Delta N)$, where the integer random variable $\Delta N$ has the same sign as $\Delta Z$, and varies within the interval $0 \leqslant|\Delta N| \leqslant|\Delta Z|$. Second, if the character value $Z+\Delta Z$ has not yet been achieved, it can be reached with probability $P_{3}$ by the change of the allelic state in $|\Delta Z-\Delta N|$ special genes. With the probability $1-P_{3}$, the phyletic lineage becomes extinct. Essentially, $P_{2}(\Delta N)$ and $P_{3}$ are conditional probabilities, since $N, Z$, and $\Delta Z$ have definite values at stage 1 , and $N, Z, \Delta Z$, and $\Delta N$ have definite values at stage 2 (we omit the corresponding symbols of the conditions). We thus assume that survival of the lineage is limited by the occurrence of favourable mutations. The dynamics of their accumulation can be described with a model of pure extinction (Feller, 1971), where the donor-of-mutation genes become extinct by mutation. The probability of exactly $m$ mutations occurring and becoming fixed in $n$ donor genes in a chosen interval $\tau$ can be determined by the following formula:

$$
P_{n}^{m}=\left(\begin{array}{c}
n \\
n-m
\end{array}\right) e^{-\lambda n}\left(e^{\lambda}-1\right)^{m}
$$

where the parameter $\lambda$ is the $\tau$ interval multiplied by the mutation rate per gene. When determining the probabilities $P_{2}$ and $P_{3}$, we used 2 parameters for the 2 stages of gene transformation, viz,

$$
\text { i) }\{Z\} \stackrel{\lambda_{1}}{\rightleftarrows}\{G-N\} \text {; ii) }\{Z\} \stackrel{\lambda_{2}}{\rightleftarrows}\{N-Z\}
$$

(the brackets refer to corresponding sets of genes); $\lambda_{1} \ll \lambda_{2}$. (The mutations of type i should occur much less frequently than those of type ii, since the former are 
connected with a change in the gene function, whereas the latter are associated with a change in gene expression.)

The probabilities $P_{2}$ and $P_{3}$ are calculated as follows. If $\Delta Z=0$ then $P_{2}(0)=$ $P_{3}=1$. If $\Delta Z>0$ then $P_{2}$ is the probability of $\Delta N$ mutations occurring in $G-N$ genes. If $\Delta Z<0$ then $P_{2}$ is the probability of $|\Delta N|$ mutations occurring in $Z$ genes, the parameter $\lambda_{1}$ being that used in [1]. The probabilities corresponding to the prohibited cases $|\Delta N|>|\Delta Z|$ are added to the probability at $\Delta N=\Delta Z$ (no excess mutations occur). $P_{3}$ is determined as the probability of no less than $|\Delta Z-\Delta N|$ mutations in $N-Z$ genes if $\Delta Z>0$, and in $Z$ genes if $\Delta Z<0$, using the parameter $\lambda_{2}$. The border conditions are: $P_{3}$ is equal to 0 providing that $Z+\Delta Z<0$ or $Z+\Delta Z>N+\Delta N$. The transitional probabilities in the chain are determined as follows:

$$
\begin{aligned}
& P\{(N, Z) \longrightarrow(N+\Delta N, Z+\Delta Z)\}=P_{1} P_{2} P_{3} \\
& P\{(N, Z) \longrightarrow(E)\}=\sum_{\Delta Z} \sum_{\Delta N} P_{1} P_{2}\left(1-P_{3}\right)
\end{aligned}
$$

The initial state of the system is $(1,1)$. The calculations were carried out for different values of $R, \lambda_{1}$ and $\lambda_{2}$.

\section{RESULTS AND DISCUSSION}

A stationary regime has been shown to exist for the considered distribution of conditional probabilities for the system to be in states $(N, Z)$ provided that it is not in state $(E)$. Figure 1 shows the dynamics of the marginal distributions of the character value $Z$ and the number of special genes $N$. The distribution of the character values is initially narrow and skewed, and then widens and becomes symmetrical, approaching the stationary distribution (fig 1a). Figure 2 shows the relationship between the mean character value $\bar{Z}$ and its standard deviation $\sigma_{Z}$, which approaches linearity. The distribution of the number of special genes $N$ behaves differently (fig 1b). The mean special gene number $\bar{N}$ grows asymptotically with the mean character value, whereas the standard deviation $\sigma_{N}$ stabilizes much sooner (fig 3), after which the shape of the distribution does not change significantly. The pattern of the distributions, the linear relationship between $\bar{Z}$ and $\sigma_{Z}$, the parallel growth of $\bar{N}$ and $\bar{Z}$, and the existence of a stationary distribution all appear to be stable over a wide range of variation of parameters (not shown).

The stationary regime of the system well corresponds to an equilibrium between the mobilities of the structures established in the evolution in a fluctuating environment, as predicted in the description of the model. This regime is achieved irrespectively of the initial values of $N$ and $Z$. Since the evolution of any working structure starts from a primitive state, we have chosen the state $(1,1)$ as initial. In the case presented, in spite of the selection having equal probability for either an increase or a decrease of the character value, the approach of the stationary distribution is accompanied by an asymptotic increase of $\bar{Z}$. This increase may correspond to the progressive development of a working structure which continues until a specific limit is achieved. Note that the standard deviation and the range of the character value also grow, $i e$ the level of interspecies variation in the character 


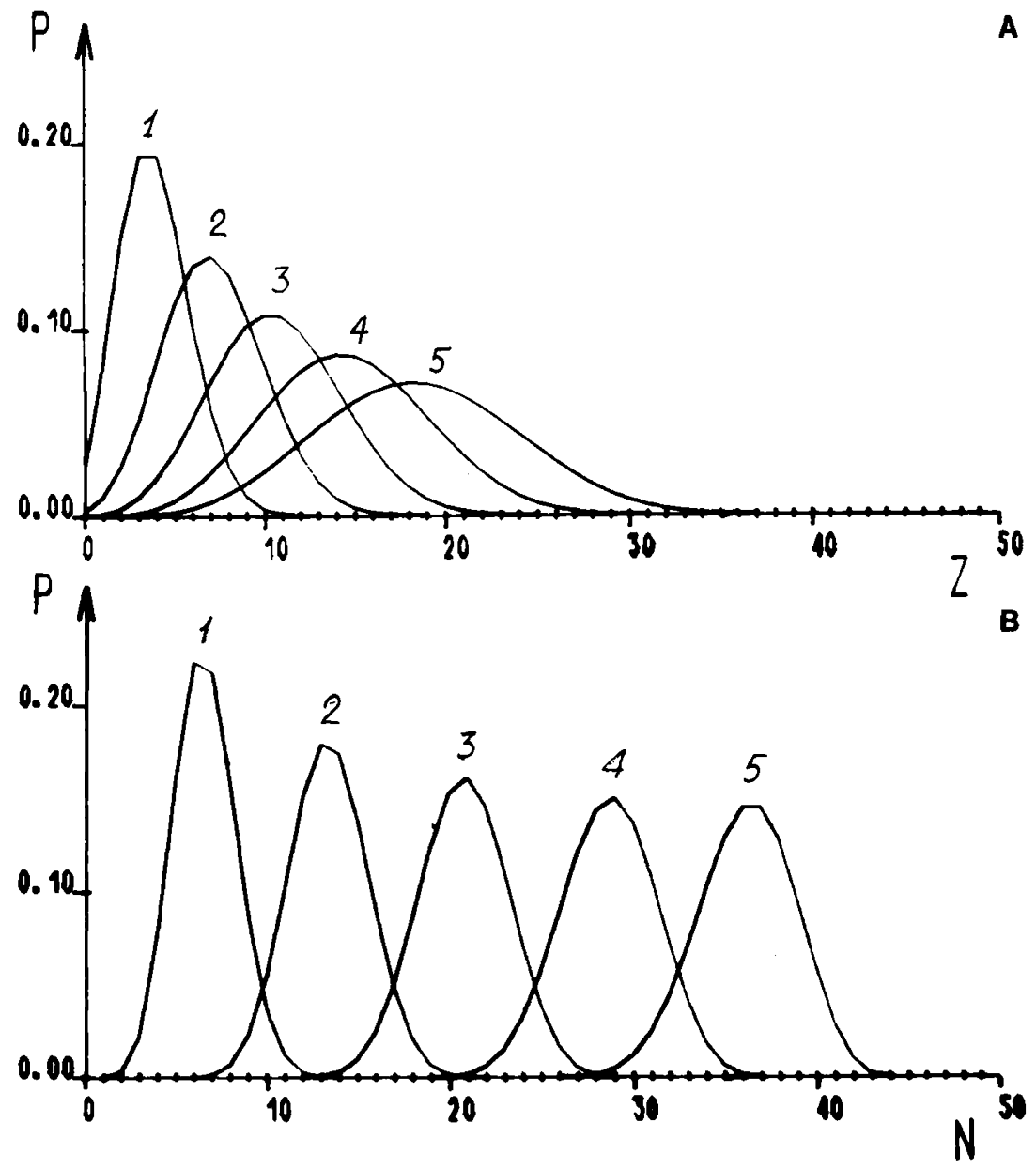

Fig 1. Dynamics of marginal (sum) distributions of character value $N(\mathrm{~A})$ and the number of special genes $Z$ (B). The distributions correspond to 20 (1), 50 (2), 100 (3), 200 (4) and 800 (5) cycles of the model; the latter is stationary. Parameter values: $\lambda_{1}=0.01$; $\lambda_{2}=0.25 ; R=50$.

value increases. Therefore, the final stationary distribution retains a large share of species with a low character value. The rise of genetic maintenance of the character, however, continues throughout the entire clade, since a parallel shift of the distribution of the $N$ values to the right is observed. This reflects the increase in the character mobility.

The mobility of the character also depends on the mutability of the special genes, determined by the parameter $\lambda_{2}$. An increase in $\lambda_{2}$ promotes the growth of $\sigma_{Z}$ and the range of the distribution of $Z$, ie the level of interspecies variation of the character value. Simultaneously, this slightly retards the growth of the mean value of the character, since the increase in $\lambda_{2}$ decreases the probability of extinction. 
The parameter $\lambda_{1}$ determines how easily mobility itself can change. The increase in this parameter accelerates the growth of the mean character value as well as the standard deviation. The increase of $R, i e$ the range of environmental change, causes the same effect.

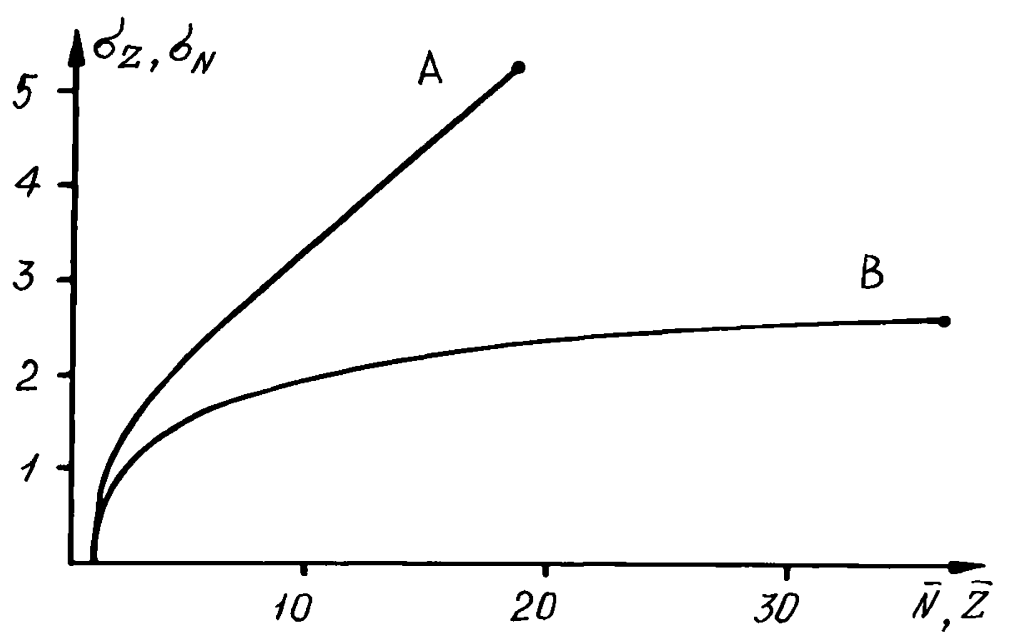

Fig 2. Relationship between the standard deviations and mean values of the distributions of character value and the number of special genes: (A) $\sigma_{Z}$ depending on $Z$; (B) $\sigma_{N}$ depending on $N$. Parameter values are the same as in figure 1.

A more realistic and sophisticated model could be constructed in which gene duplications and deletions are allowed. In this case there would be no fixed limit for the total number of genes, but rather a penalty for this number set by the increasing mutational load. The result of the model would be somewhat different, but the main qualitative feature, decelerating growth of means and variance, would remain the same.

\section{THE COMPLICATION OF THE AMMONOIDEA SUTURE}

\section{The septal suture of Ammonoidea shell}

To support the idea of mobilizing selection we need a large amount of palaeontological data on the progressive development of an easily measurable structure. The required material is readily provided by Ammonoidea. This large group of cephalopods appeared early in the Devonian, achieved a high taxonomic diversity in the Mesozoic and became extinct at the end of Cretaceous having existed more than 300 million years.

The tube of an ammonoid shell was divided by transversal septa into a large number of chambers. Each septum was in contact with the shell wall along the socalled septal suture, often an intricately curved line. Its complication, an increase in the degree of bending, was 1 of the most prominent macroevolutionary trends (Ruzhentsev, 1962). 


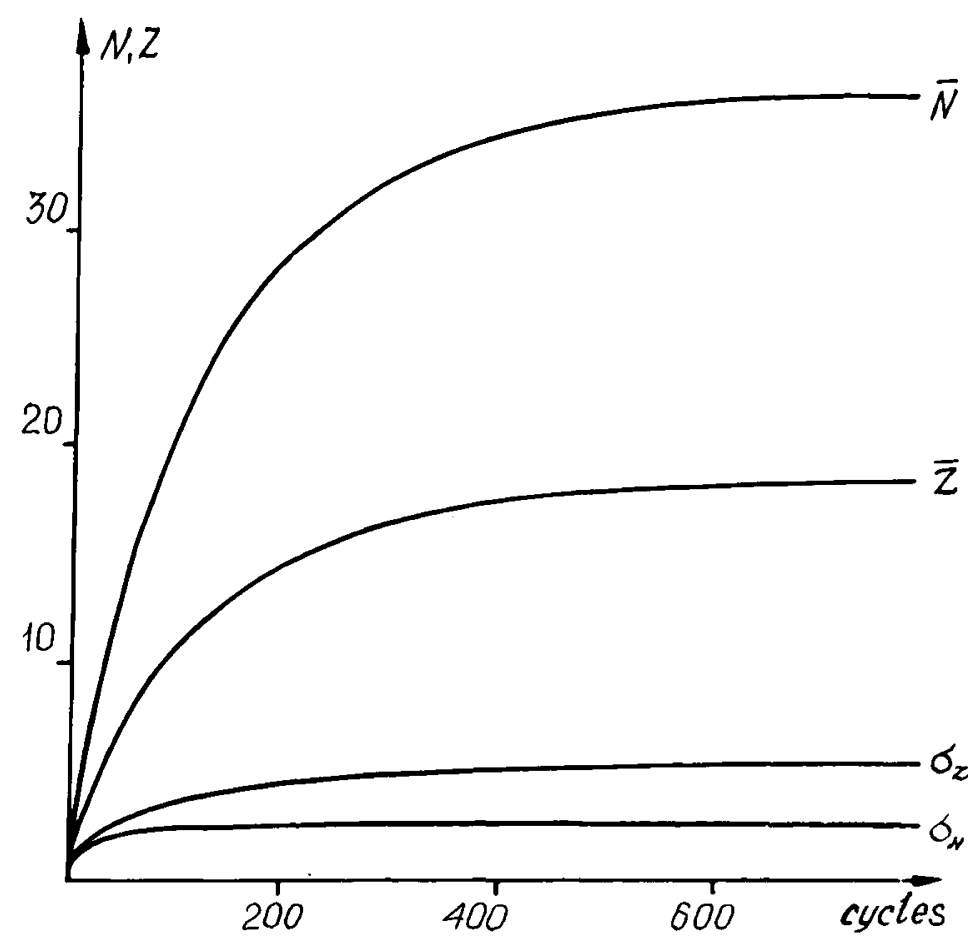

Fig 3. Change in the characteristics of the distributions in character value $Z$ and number of special genes $N$ (see text for designations) with time. Parameter values are the same as figure 1 .

In the course of individual development, the mollusc regularly constructed new chambers by adding the septa. Figure 4 shows that the complication of septal suture also occurred in ontogenesis. An impression might arise that the suture pattern was formed by repeated action of the same bending mechanism. However, there is counter-evidence.

Formally, suture pattern can be divided into elements, particular segments of the line with a single bend. Each element was formed by splitting a certain bend at 1 of the earlier stages of ontogenesis. We can trace the whole chain of successive bend splittings that lead to each adult element starting from a certain bend of primasuture (the second suture formed in development). This gives the so-called genetic formulae of the suture (Ruzhentsev, 1962). In such formulae, the lobes (bends facing the mouth of the shell by their convexity) are denoted by special symbols reflecting their origin from the primasuture lobes.

Some common modes (up to 20, see Berdnikov, 1990) of element formation can be highlighted. In the Palaeozoic Ammonoidea, only the lobes were split. Splitting of the saddles (bends facing the mouth of the shell by their concavity) occurred in the Mesozoic and, at first, affected only the slopes; later Ammonoidea learned to split the apices of saddles (Ruzhentsev, 1962). Notably, many modes of complication 


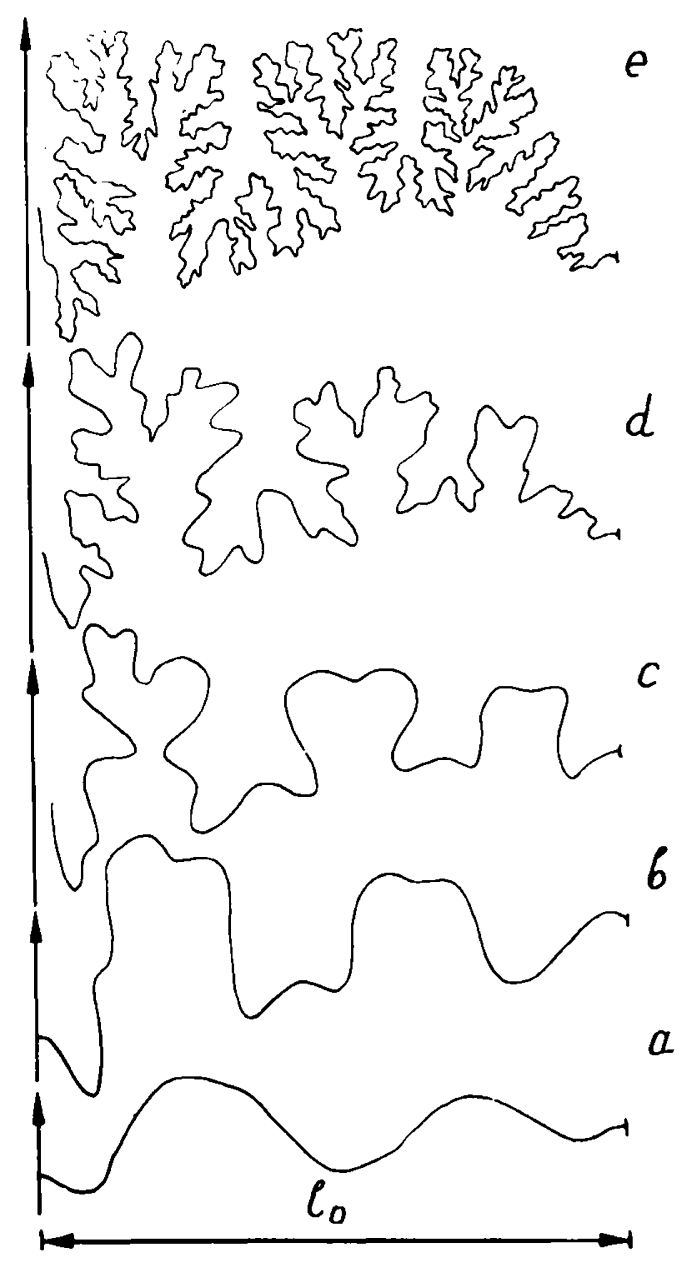

Fig 4. Complication of a suture length between the medial line and the umbilical seam in the ontogenesis of the Cretaceous ammonite Gaudryceras tenuiliratus (according to Mikhaylova (1963) with modifications). Lines (a), (b), (c), (d) and (e) refer to partitions $2,16,24,43$ and 107 of the phragmocone. The scale was normalized to the same $l_{0}$.

were acquired independently by different orders of Ammonoidea. Up to the early Jurassic an increase in the number of complication modes took place in all large taxa.

Rich palaeontological data show that the paths of suture element formation in ontogenesis of major features repeat their phylogenetic development. As a rule, the suture complication in ontogenesis of descendants was due to the addition of new elements to the suture of adult ancestors. Permanent demands of natural selection for shortening ontogenesis caused a distinct trend for shifting the stages 
characteristic of adult ancestors to the younger whorls of descendants (Ruzhentsev, 1962).

All this supposes that every element of the suture resulted from the action of a series of genes responsible for successive complication of the definite primasuture bend. Therefore, the macroevolutionary trend for complication of the suture of Ammonoidea can be interpreted as an accumulation of special genes involved in the developmental program of this structure.

\section{The data and the measurements}

We needed a measure to estimate the suture complexity. In Bactritoidea, which is thought to be ancestral to Ammonoidea, the edges of septa lacked bends. It is natural, thus, to assume that the complexity of their sutures was equal to zero. So, the measure of the complexity of the suture can be represented by the parameter $x=\ln l / l_{0}$, where $l$ stands for the suture length, and $l_{0}$ for the outline length of the cross-section of the shell-tube.

We photographed drawings of sutures at the latest stage of ontogenesis, projected the negatives on a screen and measured the suture lengths with a curvimeter. The points of intersection of the suture with the medial line and umbilical seam served as the beginning and end of the measurement, respectively. The direct distance between these points on a drawing was assumed to be $l_{0}$.

Suture drawings were taken from figures in a number of literature sources (Bogoslovskiy, 1969, 1971; Pivetau, 1952; Ruzhentsev, 1962; Ruzhentsev and Bogoslovskaya, 1971; Treatise on Invertebrate Paleontology, 1957; and some others). The values of suture complexity $(x)$ have been estimated for 1720 species of Ammonoidea. The parameter $y=\ln r$ was used as a measure of mollusc size, where $r$ is the radius of the largest individual of a species available in the literature.

\section{Results: the evolution of the suture complexity}

Figure 5 shows how the distribution of species changed with geological time. The increase in its range is striking. Over the period from the early Devonian to the late Cretaceous the range of the distribution showed a 13 -fold increase! As shown in figure 6 , over the 4 geological periods there was a steady rise in the mean suture complexity $(\bar{x})$, while in the Jurassic and the Cretaceous this mean did not change considerably. The standard deviation of suture complexity $\sigma_{x}$ reflects the interspecies variation of the trait (fig 7) and also increased up to the Jurassic. Figure 8 presents the relationship between the mean and the standard deviation of the distributions; the linear correlation coefficient $r=+0.98$ is highly significant $(P<0.005)$.

The growth of the mean complexity of the suture was interrupted by periods of stagnation and even decline. However, figure 9 demonstrates that the trend towards increasing $\bar{x}$ continued over 4 orders of Ammonoidea. Sharp changes in total $\bar{x}$ usually correspond to the periods of radical renovation of Ammonoidea fauna.

Taking into account the allometric relationship between the suture length and body size (Newell, 1949), we also studied the dynamics of shell-size distribution. Figure 6 shows a clear trend towards a decrease in the mean shell size $\bar{y}$ from the 


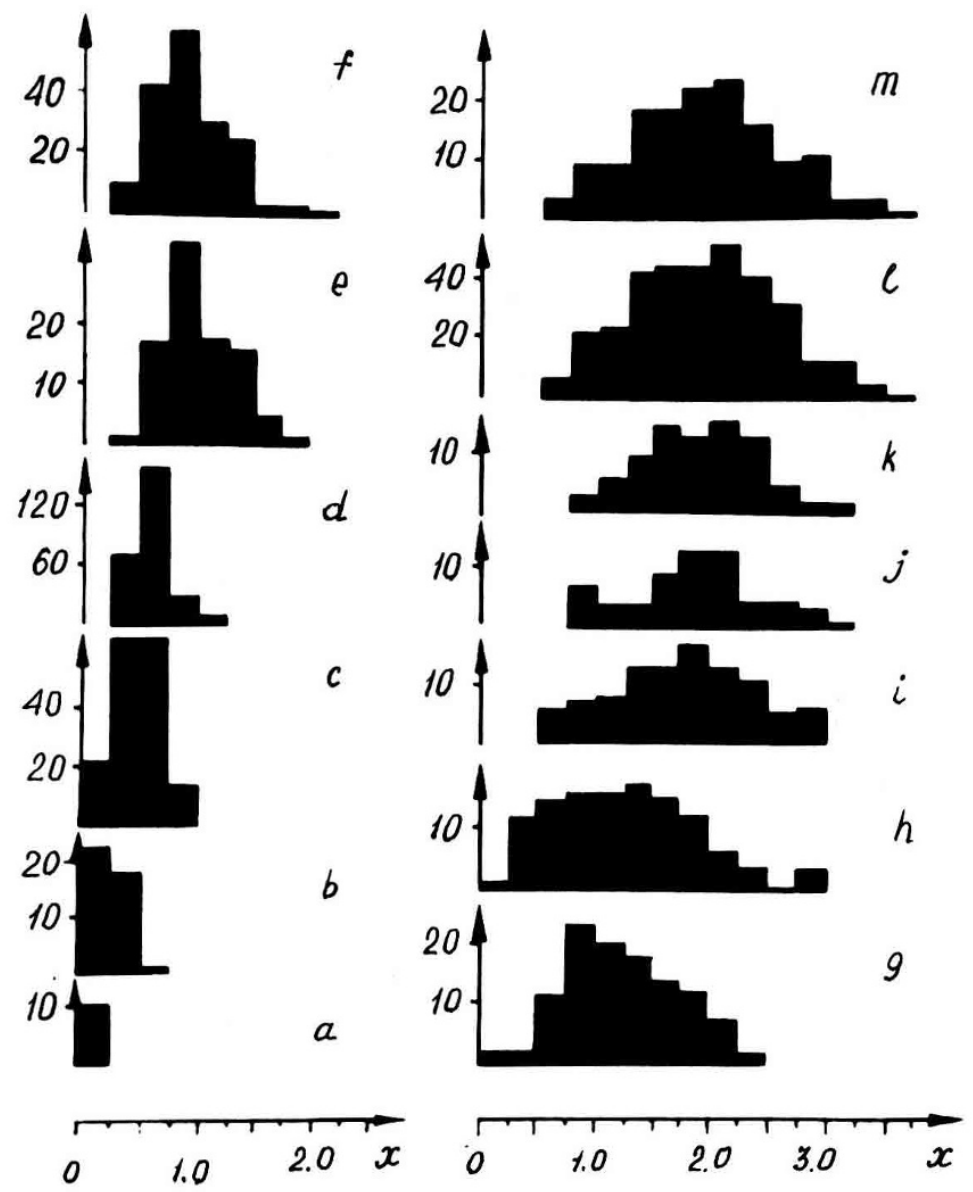

Fig 5. Distributions of suture complexity $x$ at different stages of Ammonoidea evolution: a) the early Devonian $(n=10)$; b) the middle Devonian $(n=39)$; c) the late Devonian $(n=159)$; d) the Namur age of the Carbonian $(n=249)$; e) the early Permian $(n=92)$; f) the early Triassic $(n=177)$; g) the middle Triassic $(n=111) ; \mathrm{h})$ the late Triassic $(n=121)$; i) the early Jurassic $(n=99)$; j) the middle Jurassic $(n=62)$; k) the late Jurassic $(n=75) ; 1)$ the early Cretaceous $(n=178)$; and $\mathrm{m})$ the late Cretaceous $(n=141)$.

late Devonian to the late Carbonian; later, in the early Permian, the trend reversed and the parallelism in the growth of $\bar{y}$ and $\bar{x}$ became explicit.

The interspecies variation in shell size, reflected by the standard deviation $\sigma_{y}$, did not change considerably during the evolution of Ammonoidea, while the standard deviation of the suture complexity, $\sigma_{x}$, constantly rose during the first 200 million years. The $\sigma_{x}$ was then established at a level somewhat below $\sigma_{y}$ following the dynamics of the latter. 


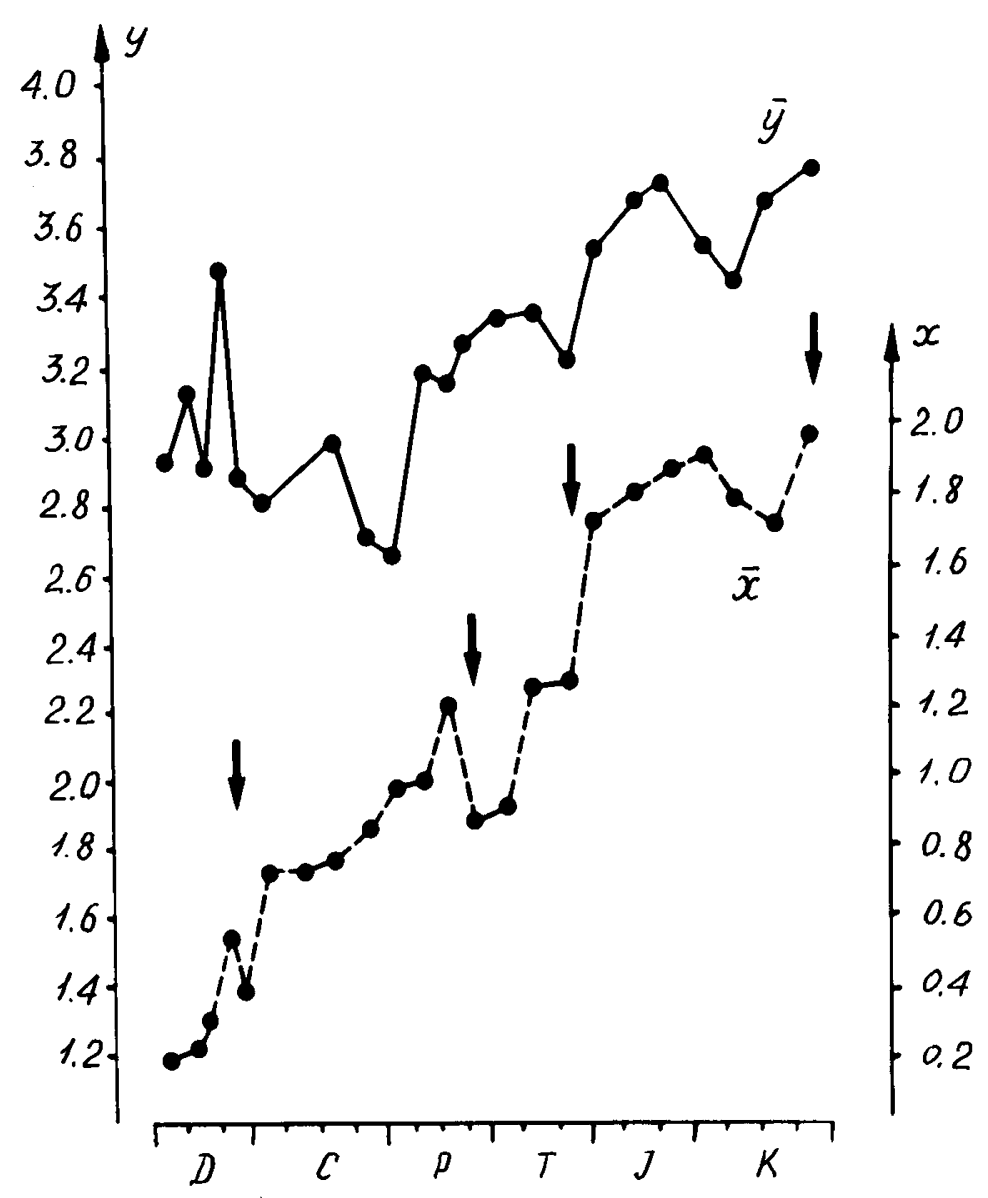

Fig 6. Dynamics of mean suture complexity of $(\bar{x})$ and mean of the maximum radius of the shell $(\bar{y})$ of Ammonoidea with geological time. Arrows indicate mass extinctions.

\section{Evolution of Ammonoid suture in terms of mobilizing selection}

It is widely accepted that the Ammonoidea shell was filled with gas and had to withstand water pressure. The folding of the septa was the method of strengthening the shell wall (Raup and Stanley, 1971). The degree of septum folding, reflected by the suture complexity, should grow faster than the shell radius due to the law of scaling (Newell, 1949). For this reason, the mobility of the suture is one of the components of the mobility of the shell size. Suture complication allows strengthening of the shell walls without a substantial increase in shell mass. Therefore, natural selection for the enlargement of the shell should also favour mutations complicating the suture. The phyletic lineages with an increased number of the special genes of the suture should have an advantage. Such lineages are capable of rapid changes in body dimensions with maintaining shell strength by a 


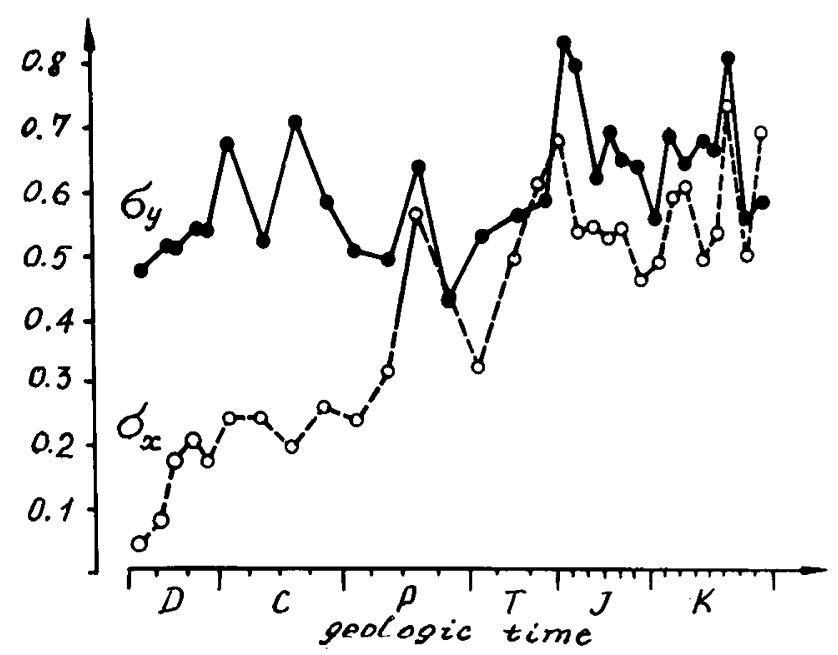

Fig 7. Dynamics of standard deviations in suture complexity $\left(\sigma_{x}\right)$ and logarithm of the radius of the shell $\left(\sigma_{y}\right)$ with geological time.

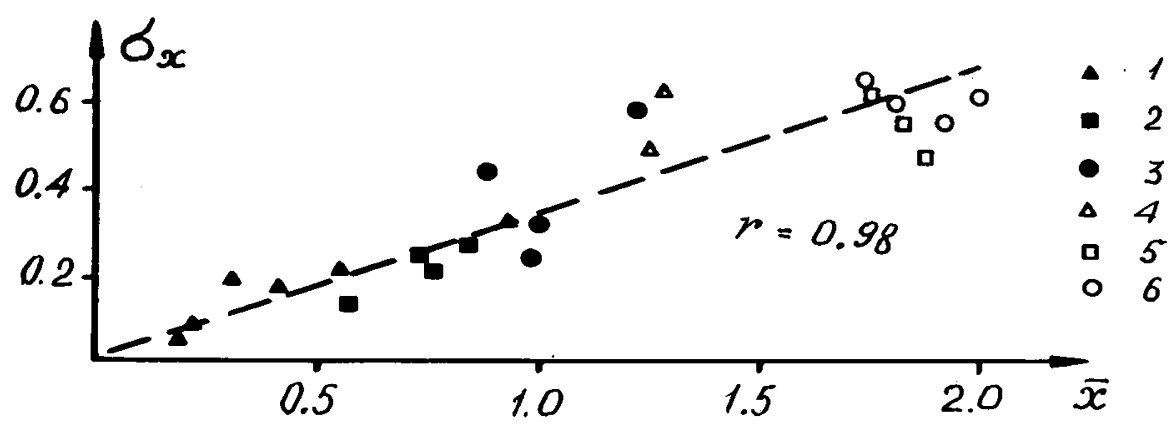

Fig 8. Relationship between the mean $(\bar{x})$ and the standard deviation $\left(\sigma_{x}\right)$ of suture complexity: 1) Devonian; 2) Carbonian; 3) Permian; 4) Triassic; 5) Jurassic; and 6) Cretaceous.

relevant suture complexity. As a result, a macroevolutionary trend should occur for the increase in the number of the suture special genes. We could trace this as a trend towards the increase in the mean value and a wide range of species distribution for the suture complexity.

This trend would evidently have reached its limit if every shift of the shell size were due to a shift of suture complexity. We can call such a suture superflexible, and designate its length by $l_{\infty}$. Let us consider the variation of its complexity. The area of the shell surface grows proportionally to the square of the radius, and the length of the superflexible suture should follow the same law. Therefore, $l_{\infty}$ 


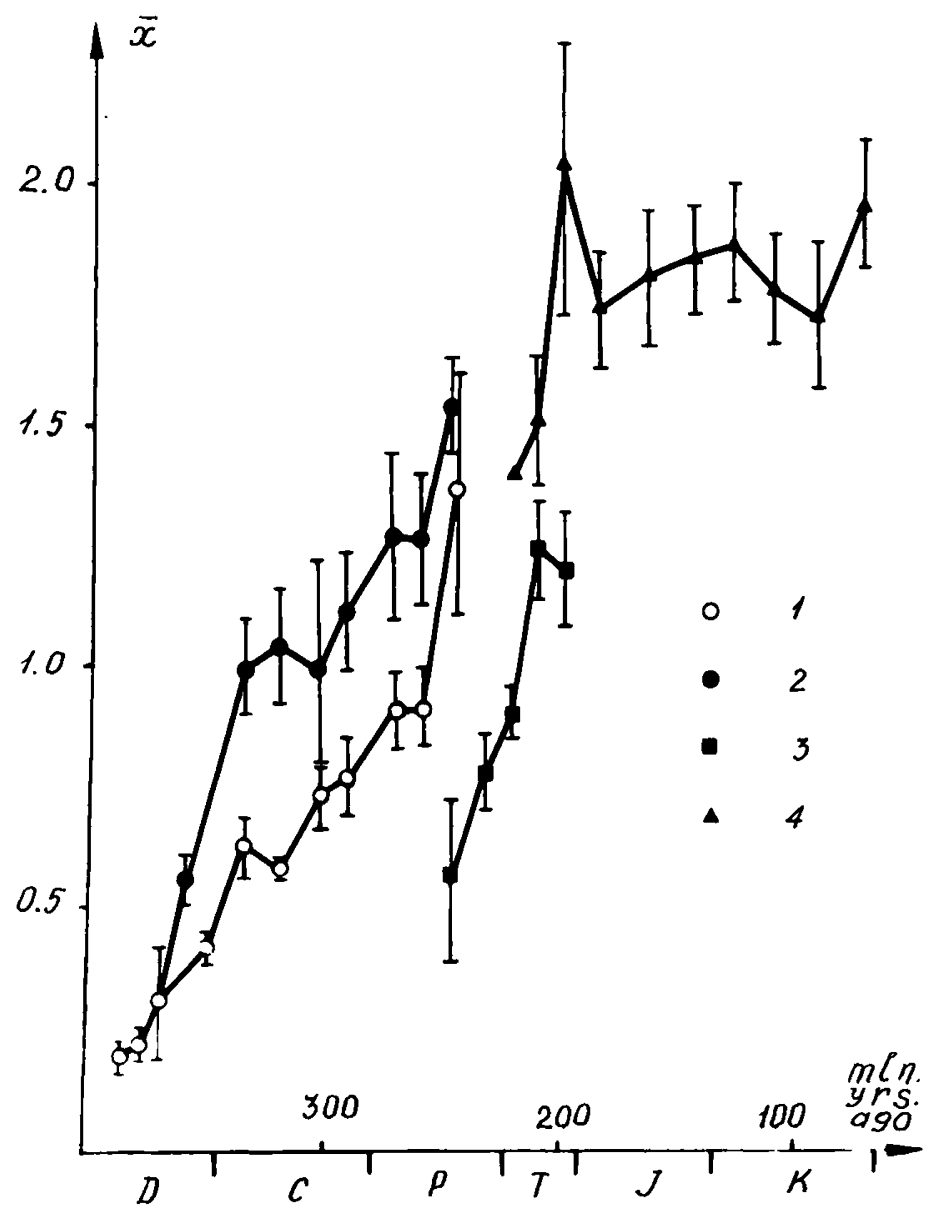

Fig 9. Dynamics of the mean suture complexity in the course of the evolution of separate orders of Ammonoidea: 1) Agoniatitida;2) Goniatitida;3) Ceratitida; and 4) Ammonitida.

should be proportional to $r^{2}$ and the ratio $l_{\infty}: l_{0}$ to $r$. Designating the coefficient of proportionality as $k$, it follows that

$$
\ln \left(l_{\infty} / l_{0}\right)=\ln r+\ln k
$$

The left-hand side of equation [4] contains the complexity of the superflexible suture $\left(x_{\infty}\right)$ while $\ln k$ in the right-hand side is constant. Since the variation of a constant is zero, the variation of the superflexible suture complexity should be equal to that of $y=\ln r$.

Our data (fig 7) show that, even in the Jurassic and the Cretaceous periods, Ammonoidea did not reach such a limit of suture mobility. The standard deviation 
of suture complexity $\left(\sigma_{x}\right)$ was steadily lower that of the shell radius logarithm $\left(\sigma_{y}\right)$, indicating that some environmental demands appear to have been satisfied by other means. Nevertheless, a concerted change of $\sigma_{x}$ and $\sigma_{y}$ during the last 130 million years of ammonoid history indicates that the capacity of the genetic system that mobilized the suture was practically exhausted.

The evolution of the Ammonoidea suture complexity well reproduces the results of the model presented. In the course of evolution, the distribution of the suture complexity widened its range by extending its maximum value and was symmetrized, tending towards the stationary distribution of the Jurassic and the Cretaceous periods. Even the relationship between the mean and the standard deviation of suture complexity approached linearity. This might indicate that the basic principles of our model fit real macroevolutionary processes.

\section{ACKNOWLEDGMENTS}

The authors express their gratitude to VV Dubatolov, for cooperation on the work on Ammonoidea, and to VS Bogdanova for helpful discussions and critical reading of the manuscript.

\section{REFERENCES}

Berdnikov VA (1990) The Basic Factors of Macroevolution (in Russian). Nauka, Novosibirsk

Berdnikov VA (1991) Evolution and Progress (in Russian). Nauka, Novosibirsk

Bogoslovskiy BI (1969) Devonian Ammonoideas. I Agoniatites (in Russian). Nauka, Moscow

Bogoslovskiy BI (1971) Devonian Ammonoideas. II. Agoniatites (in Russian). Nauka, Moscow

Cisne JL (1974) Evolution of the world fauma of aquatic free-living arthropods. Evolution 28, 337-366

Crow JF, Simmons MJ (1983) The mutation load in Drosophila. The Genetics and Biology of Drosophila. Vol. 3c Acad. Press, London, 1-35

Feller V (1971) An Introduction to Probability Theory and Its Applications. Vol 1 John Wiley \& Sons, Inc., NY

Giller PS (1984) Community Structure and the Niche. Chapman and Hall, London Grant V (1985) The Evolutionary Process. A Critical Review of Evolutionary Theory. Columbia Univ Press, NY

Kimura M (1983) The Neutral Theory of Molecular Evolution. Camb Univ Press, Cambridge

Kondrashov AS (1988) Deleterious mutations and the evolution of sexual reproduction. Nature (Lond) 336, 435-440

Mather K, Jinks DL (1982) Biometrical Genetics: The Study of Continuous Variation. 3rd ed. Chapman and Hall, London

Mikhaylova IA (1963) Systematics and Phylogeny of Cretaceous Ammonoideas (in Russian). Nauka, Moscow 
Newell ND (1949) Phyletic size increase - an important trend illustrated by fossil invertebrates. Evolution 3, 103-124

Pivetau J (1952) Traité de Paléontologie, II. Masson, Paris

Raup D, Stanley S (1971) Principes of Paleontology. WF Freeman \& Co, San Francisco

Ruzhentsev VE (1962) Ammonoidea. II. Bases of Paleontology. MolluscaCephalopoda (in Russian). Ed. of Acad Sci USSR, Moscow

Ruzhentsev VE, Bogoslovskaya MF (1971) The Namur Stage in Ammonoidea Evolution: Ammonoidea of Early Namur (in Russian). Nauka, Moscow

Ruzhentsev VE, Bogoslovskaya MF (1978) The Namur Stage in Ammonoidea Evolution: Ammonoidea of Late Namur (in Russian), Nauka, Moscow

Schmidt-Nielsen K (1984) Scaling. Why is Animal Size So Important? Camb Univ Press, Cambridge

Simpson GG (1953) The Major Features of Evolution. Columbia Univ Press, NY

Stanley SM (1973) An explanation for Cope's rule. Evolution 27, 1-26

Sturtevant AH (1965) A History of Genetics. Harper \& Row, NY

Treatise on Invertebrate Paleontology, part L. Mollusca 4, Cephalopoda, Ammonoidea. (1957) Univ of Kansas Press, Kansas City

Wistow GL, Piatigorsky J (1987) Recruitment of enzymes as lens structural proteins. Science 236, 1554-1556

Wright S (1984) Evolution and the Genetics of Populations. Vol 1: Genetic and Biometric Foundations. Univ of Chicago Press, Chicago 